\title{
Staging Incomplete
}

National Cancer Institute

\section{Source}

National Cancer Institute. Staging Incomplete. NCI Thesaurus. Code C159243.

A lack of clinical and/or pathology information to assign a stage conclusively. 